\title{
Efficient Protein Transfection by Swarms of Chemically Powered Plasmonic
}

\section{Virus-Sized Nanorobots}

Alzbeta Ressnerova ${ }^{1,2}$, Filip Novotny ${ }^{3}$, Hana Michalkova ${ }^{1,2}$, Martin Pumera ${ }^{3,4,5,6,7^{*}}$, Vojtech Adam $^{1,2 \dagger}$, Zbynek Heger $^{1,2 * *}$

${ }^{1}$ Department of Chemistry and Biochemistry, Mendel University in Brno, Zemedelska 1, CZ61300 Brno, Czech Republic

${ }^{2}$ Central European Institute of Technology, Brno University of Technology, Purkynova 123, CZ-612 00 Brno, Czech Republic

${ }^{3}$ Center for Advanced Functional Nanorobots, Department of Inorganic Chemistry, University of Chemistry and Technology, Technicka 5, CZ-166 28 Prague, Czech Republic

${ }^{4}$ Center for Nanorobotics and Machine Intelligence, Department of Food Technology, Mendel

University in Brno, Zemedelska 1, CZ-613 00 Brno, Czech Republic

${ }^{5}$ Department of Medical Research, China Medical University Hospital, China Medical University, No. 91 Hsueh-Shih Road, 40402 Taichung, Taiwan

${ }^{6}$ Department of Chemical and Biomolecular Engineering, Yonsei University, 50 Yonsei-ro, Seodaemun-gu, 03722 Seoul, Korea

${ }^{7}$ Future Energy and Innovation Lab, Central European Institute of Technology, Brno University of Technology, Purkynova 123, CZ-612 00 Brno, Czech Republic

\section{Corresponding Authors}


*Prof. Martin Pumera, Center for Advanced Functional Nanorobots, University of Chemistry and Technology, E-mail: martin.pumera@ ceitec.vutbr.cz

†Prof. Vojtech Adam, Mendel University in Brno, E-mail: vojtech.adam@mendelu.cz **Dr. Zbynek Heger, Central European Institute of Technology, E-mail: zbynek.heger@ceitec.vutbr.cz

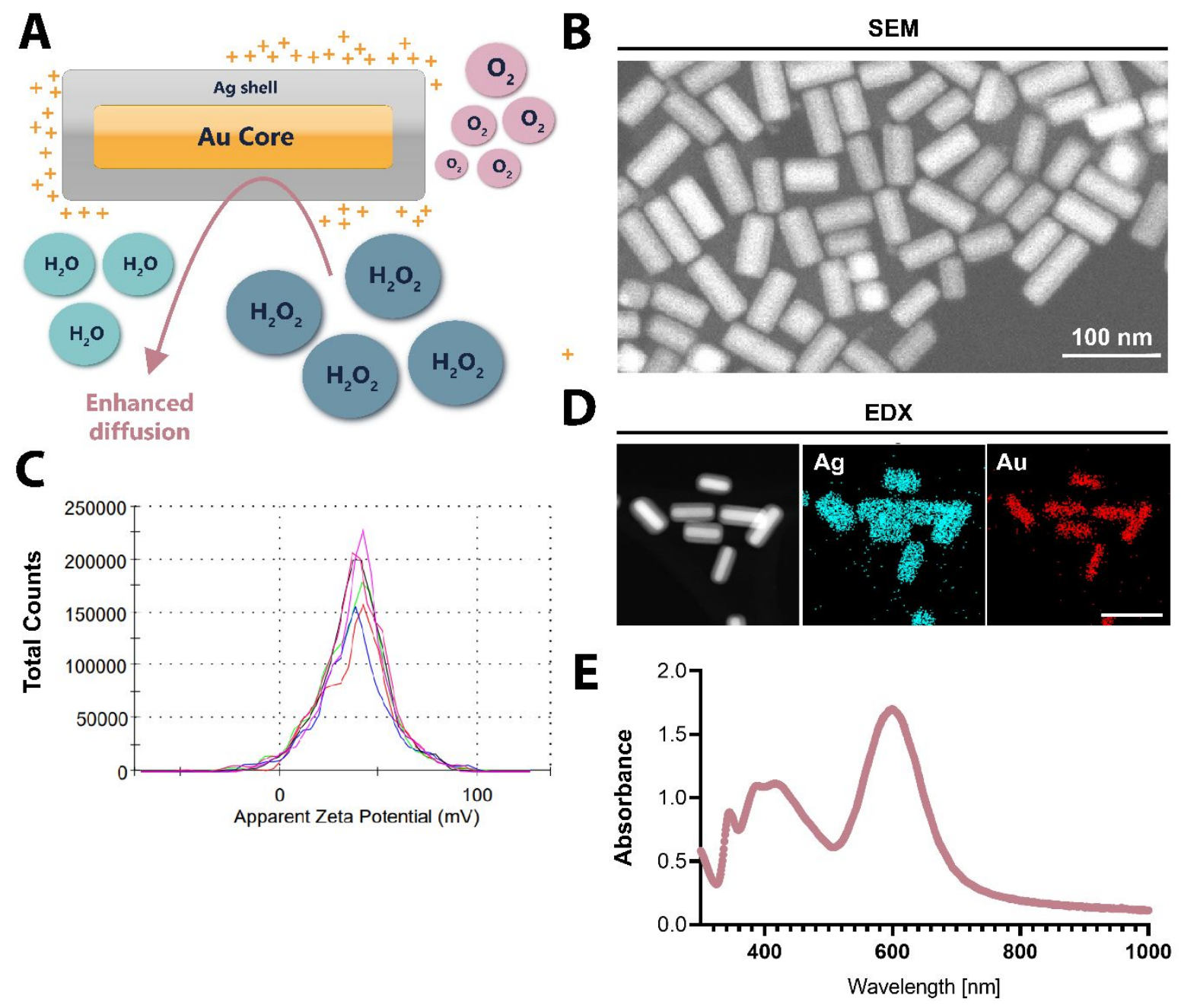

Supplementary Figure 1: Physico-chemical characterization of the AuAg-nanorobots. (A) Schematic depiction of Hydrogen peroxide-propelled rectangular cationic AuAg-nanorobots used in this study. (B) SEM micrograph of the AuAg-nanorobots taken using HADF transmitted electron detector showing the contrast between the Au core and the Ag shell. (C) $\zeta$-potential measurement of the AuAg-nanorobots in $1 \mathrm{mM} \mathrm{KCl}$ showing stable colloid dispersion with $\zeta$ potential maximum $38.8 \mathrm{mV}$. (D) EDX elemental mapping of the AuAg-nanorobots confirming 
the assembly of AuAg-nanorobots composed of Au core and Ag shell. Scale bar, $100 \mathrm{~nm} .(\mathbf{E})$ UV-Vis spectrum of the AuAg-nanorobots in aqueous solution showing the characteristic localized plasmon resonance absorption bands of AuAg-nanorobots plasmonic nanostructures. 


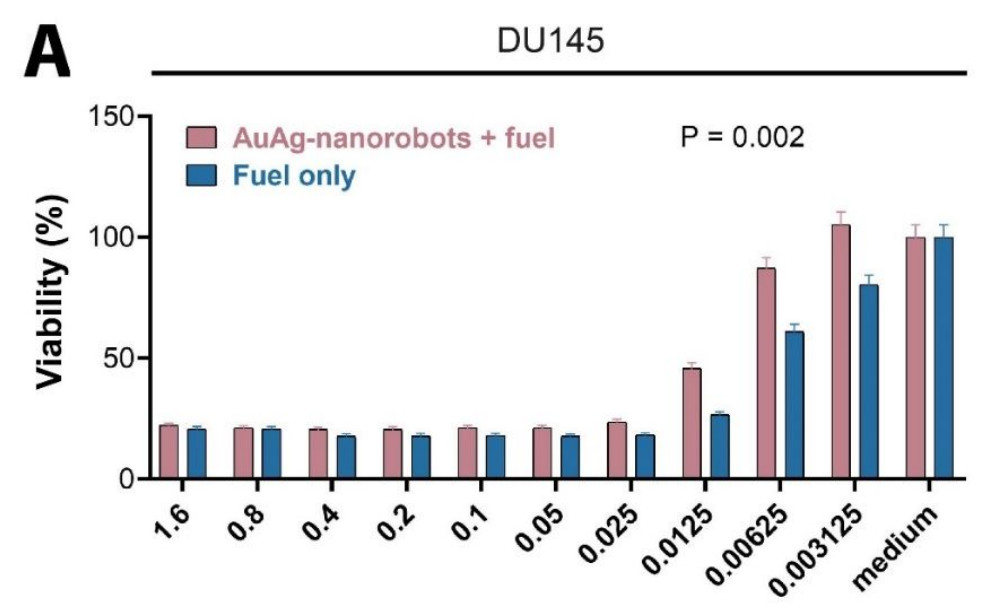

Fuel concentrations (\%)

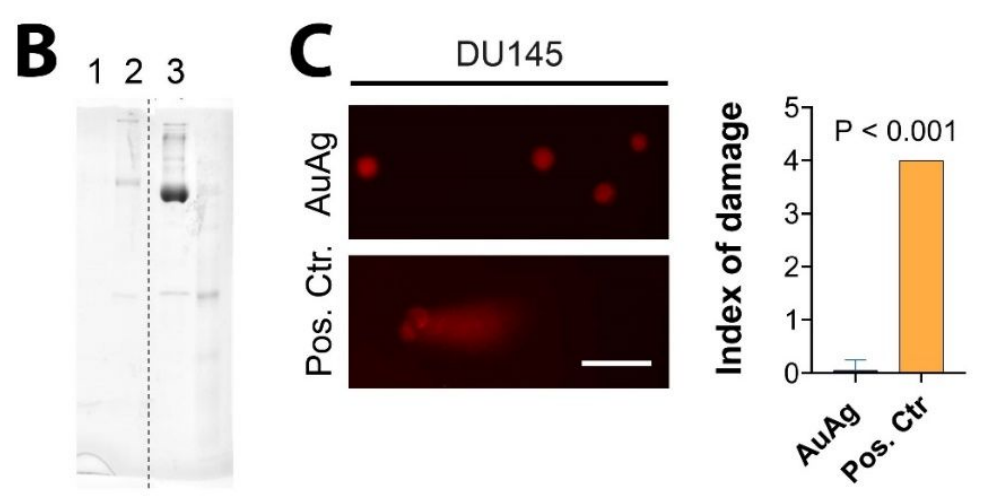

Supplementary Figure 2: Cytotoxicity screening to assess biocompatibility of AuAgnanorobots. (A) MTT assay showing significantly higher viability of cells treated with constant concentration of AuAg-nanorobots $\left(1.6 \times 10^{7}\right)$ and various concentrations of hydrogen peroxide compared to hydrogen peroxide treatment alone (Wilcoxon, $\mathrm{P}=0.002$ ). Error bars represent standard error. (B) Hard protein corona-formation assay indicating neglectable formation of protein corona on AuAg-nanorobots after incubation with human plasma. 1- AuAg-nanorobots alone, 2- AuAg-nanorobots with human plasma, 3- plasma (diluted 1000×). Dashed line indicates cropping of a sample-free are. (C) SCGE demonstrating that AuAg-nanorobots do not induce genotoxic damage (Mann-Whitney, $\mathrm{P}<0.001$ ). Scale bar, $20 \mu \mathrm{m}$. Data are presented as mean $\pm \mathrm{SD}$ 


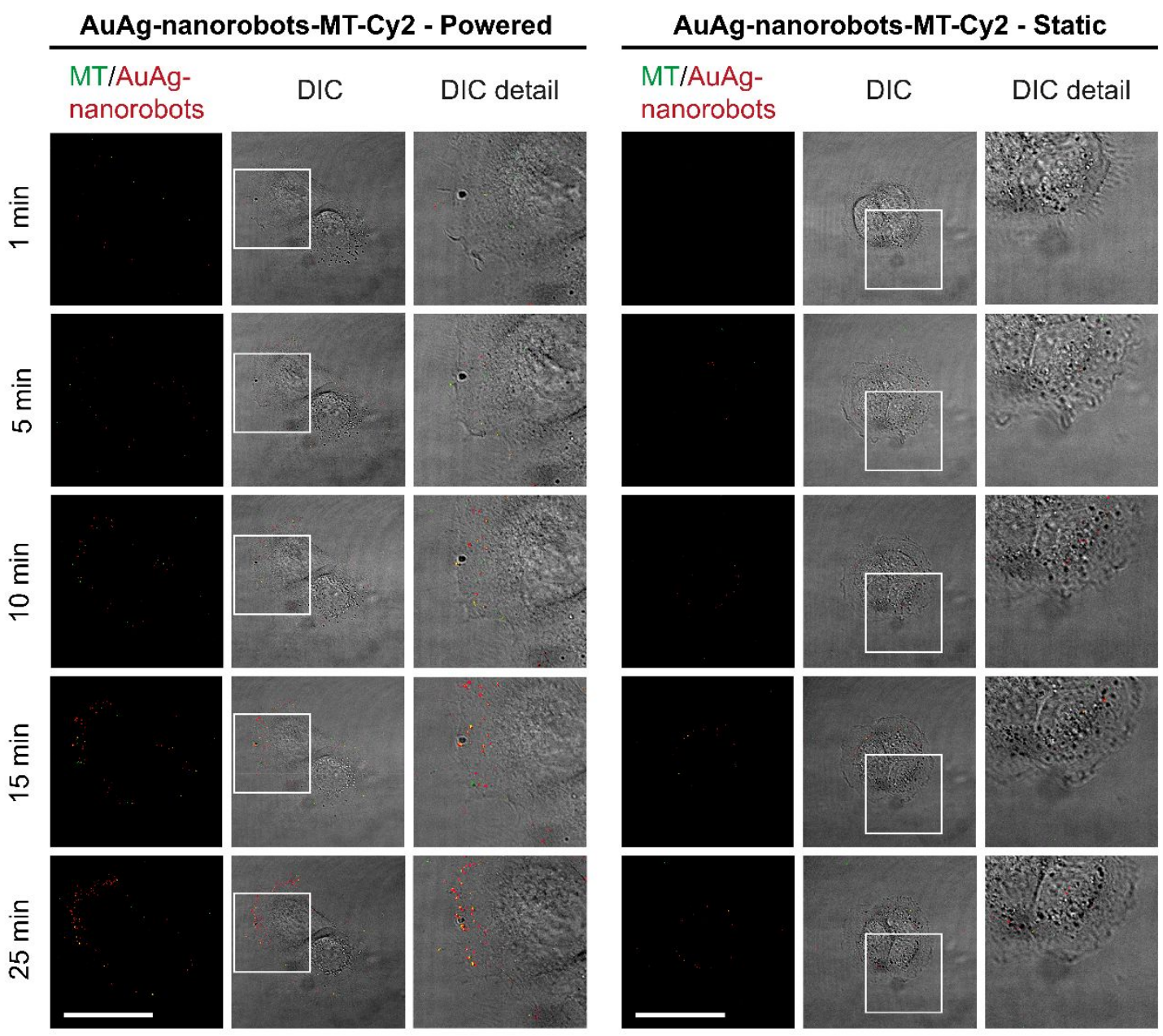

Supplementary Figure 3: Representative micrographs showing spatiotemporal kinetics of uptake of AuAg-nanorobots-MT-Cy2 (2 × $10^{8}$ of AuAg-nanorobots $)$ in fueled- and non-fueled medium in various time points (up to $25 \mathrm{~min}$ ). Scale bar, $20 \mu \mathrm{m}$. 